\title{
Utilização de água residuária de origem doméstica na agricultura: Estudo do estado nutricional do cafeeiro ${ }^{1}$
}

\author{
Salomão de S. Medeiros ${ }^{2}$, Antônio A. Soares ${ }^{3}$, Paulo A. Ferreira ${ }^{3}$, Júlio C. L. Neves $^{3}$ \& José A. de Souza ${ }^{3}$
}

\section{RESUMO}

Neste trabalho o objetivo principal foi investigar o estado nutricional do cafeeiro em resposta à aplicação de água residuária filtrada de origem doméstica, como fonte de nutrientes e comparar os resultados com os obtidos com o manejo convencional. O experimento foi implantado na Unidade Piloto de Tratamento de Água Residuária e Agricultura Irrigada, localizada na Universidade Federal de Viçosa - UFV. O delineamento experimental constituiu-se de 18 unidades experimentais, cada uma composta de oito plantas. O experimento foi montado no delineamento em blocos casualizados (linhas de plantio) com três repetições, cujo resultados permitiram concluir que a adoção do manejo com água residuária foi mais efetivo na melhoria do estado nutricional do cafeeiro que o manejo convencional.

Palavras-chave: fertirrigação, café, reúso

\section{Use of wastewater of domestic origin in agriculture: A study of nutritional status of coffee}

\begin{abstract}
The objective of this work was to investigate nutritional status of coffee in response to application of filtered domestic wastewater and to compare the results with conventional agricultural management. The experiment was carried out at the Sewer Treatment Pilot Plant (EPTE), DEA/UFV. The experimental design consisted of 18 plots, each with eight plants. The treatments were distributed in randomized blocks design (planting lines) with three repetitions. According to the results, it may be concluded that the adoption of the management with wasterwater was more effective to improve nutritional status of coffee in comparison to conventional management.
\end{abstract}

Key words: fertirrigation, coffee, reuse

\footnotetext{
1 Parte da Tese de Doutorado do primeiro autor, apresentada à Universidade Federal de Viçosa - UFV

2 CODEVASF/2a SR, CEP 47600-000, Bom Jesus da Lapa, BA. Fone: (77) 3481-8020. E-mail: salommao@gmail.com

3 DEA/UFV, CEP 36571-000, Viçosa, MG. Fone: (31) 3899-1908. E-mail: aasoares@ufv.br; pafonso@ufv.br
} 


\section{INTRODUÇÃO}

Em determinados países, razões culturais favorecem a aplicação de resíduos ao solo, ao invés de descarregá-los nos corpos d’água (Cameron et al., 1997); em outros, como o Brasil, há falta de tradição na reciclagem dos resíduos gerados, particularmente do efluente de esgoto; todavia, nos últimos anos a aplicação de resíduos orgânicos na agricultura tem recebido atenção considerável devido ao, aumento sempre crescente, do requerimento de energia para produção de fertilizantes minerais e em virtude dos custos e problemas ambientais associados aos métodos alternativos de disposição de resíduos (Chae \& Tabatabai, 1986).

Segundo van der Hoek et al. (2002), as maiores vantagens do aproveitamento da água residuária, são: conservação da água disponível, sua grande disponibilidade, possibilidade do aporte e a reciclagem de nutrientes (reduzindo a necessidade de fertilizantes químicos) e concorrer para a preservação do meio ambiente.

Estudos efetuados em diversos países demonstraram que a produtividade agrícola aumenta significativamente em áreas fertirrigadas com águas residuárias de origem doméstica, desde que sejam adequadamente manejadas.

Segundo Feigin et al. (1978) a quantidade de N adicionado ao solo por meio da irrigação com água residuária de origem doméstica, pode ser similar, ou até mesmo exceder, a quantidade aplicada via fertilização nitrogenada recomendada durante períodos de tempo similares.

Al-Jaloud et al. (1995) verificaram que as concentrações de N, P, K, Mg, Na, Cu, Mn, Mo e Zn nas folhas de milho aumentaram mediante a fertirrigação com efluente de estação de tratamento e, ainda, que 82 a 99\% da variabilidade na concentração desses minerais nas plantas decorreram das lâminas aplicadas. Em outro experimento com a cultura do sorgo, esses autores observaram, aumento nas concentrações foliares de $\mathrm{N}$, Ca, Mg e $\mathrm{Na}$, mediante a fertirrigação com efluente de estação de tratamento e diminuição nas concentrações de P e K.

Segundo Rezende (2003) a aplicação de efluente tratado (proveniente do processo de fabricação de celulose) influenciou na produção de biomassa e no crescimento das plantas de eucalipto, após 240 dias de aplicação, e o aspecto nutricional das plantas foi compatível com valores de referência de níveis críticos e faixa de suficiência para a cultura do eucalipto.

A literatura relata que as frutíferas vêm mostrando resultados satisfatórios quando fertirrigadas com efluente de estação de tratamento (Maurer \& Davies, 1993; Lurie et al., 1996), sem maiores efeitos deletérios às plantas e ao ambiente e, ainda, tem havido economia de fertilizantes convencionais (Fitzpatrick et al., 1986). Johns \& McConchie (1994a) verificaram que a cultura da banana foi $10 \%$ mais produtiva mediante fertirrigação com água residuária. Ainda neste experimento se observou que a aplicação de $600 \mathrm{~mm}$ de efluente de estação de tratamento atendeu a $20 \%$ do total de $\mathrm{N}$ requerido pela bananeira sem, no entanto, ocasionar dano ambiental (Johns \& McConchie, 1994b). Neste sentido, o presente trabalho, objetivou-se investigar o estado nutricional do cafeeiro, em resposta à fertirrigação com água residuária filtrada de origem doméstica e comparar os resultados com aqueles obtidos com o manejo convencional.

\section{MATERIAL E MÉTODOS}

O experimento foi realizado na Unidade Piloto de Tratamento de Água Residuária e Agricultura Irrigada, localizada na Universidade Federal de Viçosa - UFV, pertencente ao Departamento de Engenharia Agrícola - DEA. A Unidade Piloto é constituída de uma estação elevatória e uma unidade de tratamento de água residuária de origem doméstica (abastecida pelo esgoto proveniente do condomínio Bosque Acamari) conjugada a um sistema de irrigação localizada por gotejamento que possibilita a aplicação do efluente em uma área de 0,14 ha de cafeeiro (variedade Catuaí) com três anos de idade, cujo espaçamento é de 2,5 m entre linhas de plantio e $0,75 \mathrm{~m}$ entre plantas. A classificação do solo da área experimental, segundo Vieira (2003), é um Cambissolo Háplico Tb distrófico latossólico, dividido em cinco horizontes denominados: horizonte $\mathrm{A}$, de 0 a $0,13 \mathrm{~m}$ de profundidade; horizonte $\mathrm{AB}$ de 0,13 a $0,26 \mathrm{~m}$ de profundidade; horizonte $\mathrm{BA}$, de 0,26 a $0,48 \mathrm{~m}$; horizonte $\mathrm{B}_{1}$, de 0,48 a $0,75 \mathrm{~m}$ e o horizonte $\mathrm{B}_{2}$ de 0,75 a $1,00 \mathrm{~m}$. Apresentam-se, na Tabela 1, as características químicas do solo para a condição inicial e durante o período de monitoramento.

Na realização do experimento usou-se apenas parte da Unidade Piloto, na qual se montou uma infra-estrutura para aplicação da água residuária bruta, proveniente do esgoto doméstico (Figura 1). A infra-estrutura é formada de uma linha de derivação, que capta a água residuária bruta da adutora e a conduz a um filtro de areia, para ser filtrada; após a filtragem, a água residuária é armazenada em um tanque com capacidade de $2.500 \mathrm{~L}$, o qual possui um sistema de motobomba acoplado, possibilitando sua aplicação através de um sistema de irrigação por gotejamento, após filtrada novamente por um filtro de disco.

O filtro de areia tem formato cilíndrico com fluxo ascendente tendo, como material filtrante, camadas sobrepostas de cascalho, areia grossa lavada, areia fina lavada, areia grossa lavada e cascalho; já o filtro de disco é de 1 " de 120 mesh com capacidade de filtragem de até 5,0 $\mathrm{m}^{3} \mathrm{~h}^{-1}$; por outro lado, o filtro de areia trabalhava com capacidade de filtragem de aproximadamente $0,2 \mathrm{~m}^{3} \mathrm{~h}^{-1}$.

Anterior à implantação desta infra-estrutura, realizaramse amostragens foliares do cafeeiro em várias plantas da área selecionada para determinação de sua condição inicial $\left(\mathrm{C}_{0}\right)$. As amostragens foliares foram realizadas coletando-se os $3^{\circ}$ e $4^{\circ}$ pares de folhas, a partir do ápice dos ramos produtivos, na altura mediana do cafeeiro. No Laboratório de Análise foliar, do Departamento de Solos, determinaram-se os teores foliares de $\mathrm{N}, \mathrm{P}, \mathrm{K}, \mathrm{Ca}, \mathrm{Mg}, \mathrm{S}$, $\mathrm{Zn}, \mathrm{Fe}, \mathrm{Mn}$, Cu e B, nas folhas do cafeeiro, segundo metodologias descritas em EMBRAPA (1997).

O delineamento experimental adotado totalizou 18 unidades 
Tabela 1. Características químicas do solo durante o período de monitoramento* em resposta à adoção do tipo de manejo

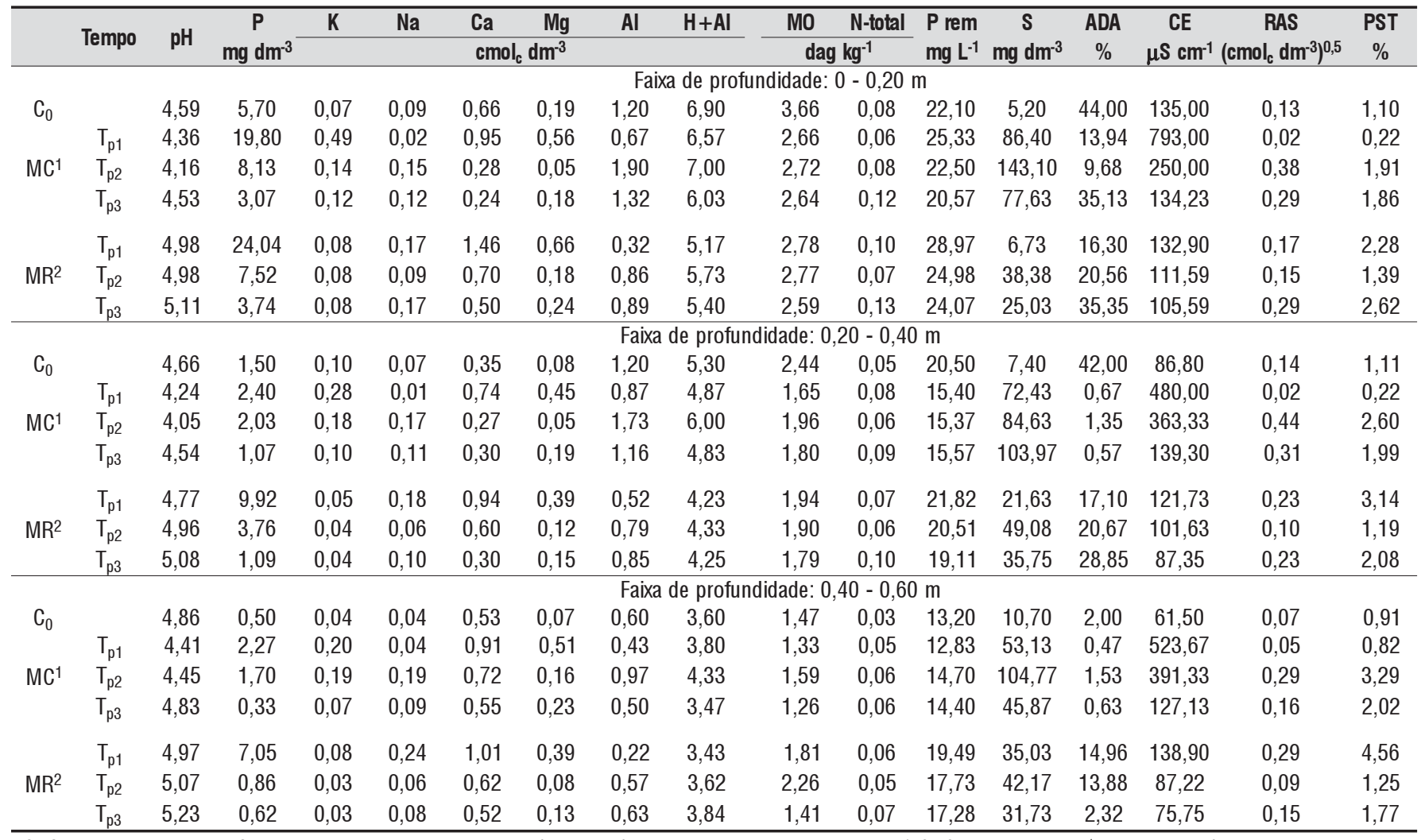

${ }^{*} \mathrm{C}_{0}$ : Condição inicial do solo; MC: Manejo convencional; MR: Manejo com água residuária; $T_{p 1}, T_{p 2}$ e $T_{p 3}$ referentes aos tempos 1,2 e 3 , respectivamente; ${ }^{1}$ Valores das variáveis no manejo convencional provêm da média das 3 repetições; ${ }^{2}$ Valores das variáveis no manejo com água residuária se originam da média geral dos tratamentos $T_{2}, T_{3}, T_{4}, T_{5}$ e $T_{6}$
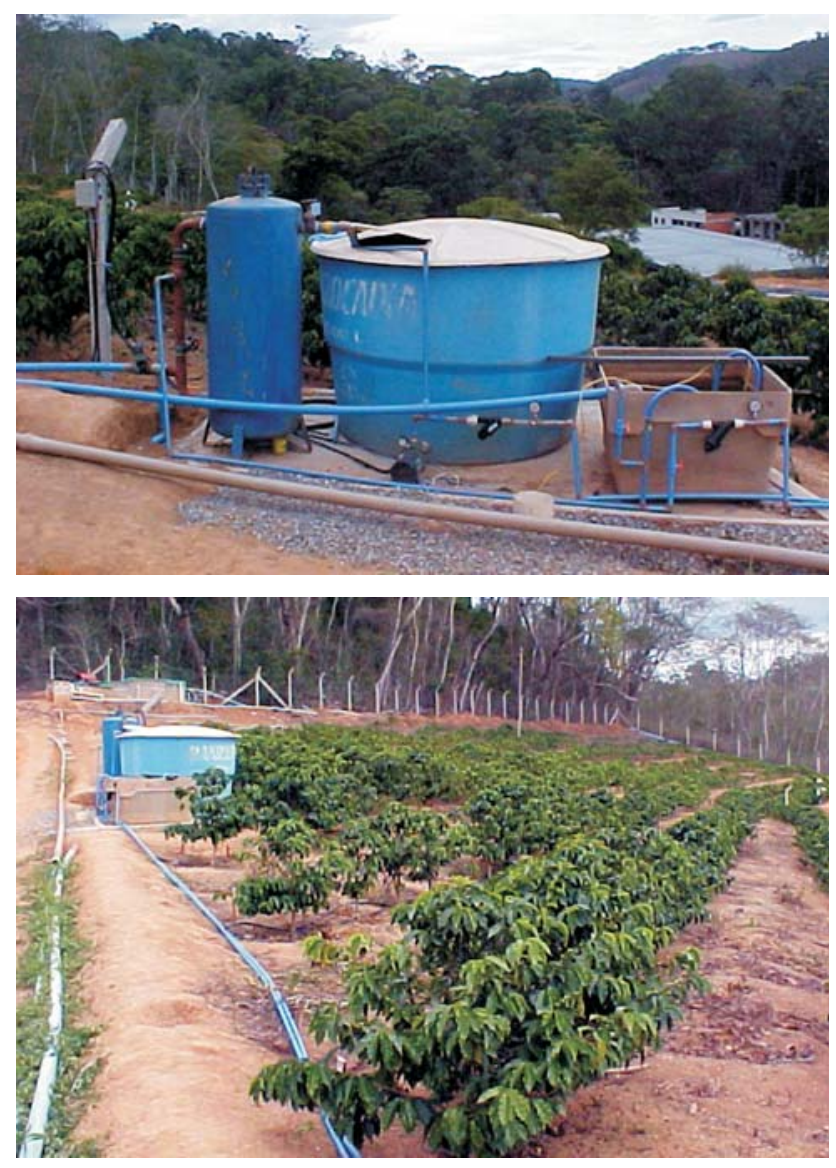

Figura 1. Infra-estrutura montada para aplicação da água residuária filtrada experimentais, com oito plantas cada uma, ocupando área de $15 \mathrm{~m}^{2}$. O experimento foi montado segundo o delineamento em blocos casualizados, tendo nos blocos (linhas de plantio) os tipos de manejos adotados (convencional - MC e com água residuária - MR, com aplicação de cinco diferentes lâminas) com três repetições.

O período de monitoramento do estado nutricional do cafeeiro foi de 270 dias realizando-se, a cada 90 dias, amostragens foliares. O experimento foi iniciado em novembro de 2003 e concluído em agosto de 2004. As amostragens foram realizadas nas quatro plantas centrais de cada unidade experimental, sendo que em cada planta foram coletados dois pares de folhas de cada lado.

O MC (tratamento $\mathrm{T}_{1}$ ), consistiu de calagem, adubação convencional e irrigação suplementar com água da represa. Realizaram-se a calagem e adubação do cafeeiro, com base na análise química do solo (Tabela 1) para condição inicial - $\mathrm{C}_{0}$, seguindo-se a recomendação da CFSEMG (1999). A calagem foi realizada com base no método da neutralização do $\mathrm{Al}^{3+}$ e da elevação dos teores de $\mathrm{Ca}^{2+}$ e $\mathrm{Mg}^{2+}$, aplicandolhe de uma só vez, $1.000 \mathrm{~kg} \mathrm{ha}^{-1}$ ano $^{-1}$ de calcário na superfície e linha contínua, abaixo da saia do cafeeiro. Na adubação convencional foram aplicados $50 \mathrm{~kg} \mathrm{ha}^{-1} \mathrm{ano}^{-1}$ de $\mathrm{P}_{2} \mathrm{O}_{5}$, tomando-se por base as concentrações iniciais de $\mathrm{P}$ disponível no solo e a de $\mathrm{P}$ remanescente (Tabela 1 ), considerandose uma produtividade 31 a $40 \mathrm{sc} \mathrm{ha}{ }^{-1}$. A fonte de $\mathrm{P}_{2} \mathrm{O}_{5}$ utilizada foi o superfosfato simples, aplicado de uma só vez; por outro lado, a adubação com $\mathrm{N}$ e $\mathrm{K}^{+}$foi realizada aplicando-se 
$300 \mathrm{~kg} \mathrm{ha}^{-1} \mathrm{ano}^{-1}$ de $\mathrm{N}$ e de $\mathrm{K}_{2} \mathrm{O}$, com base na concentração de $\mathrm{N}$ nas folhas e na concentração de $\mathrm{K}^{+}$no solo. A aplicação desses nutrientes foi feita a lanço, abaixo da saia do cafeeiro e parcelada três vezes, com intervalo de 30 dias. A fonte de $\mathrm{N}$ utilizada foi o sulfato de amônia e a de K, o cloreto de potássio.

A irrigação foi realizada no MC, com base na evapotranspiração do cafeeiro - $\mathrm{Et}_{\mathrm{c}}$ (Eq. 1), sendo que, na estimativa da evapotranspiração de referência - $\mathrm{Et}_{0}$, utilizouse o modelo proposto por Penman-Monteith (Allen et al., 1998), onde instalou-se uma estação climatológica na área experimental, para monitoramento das condições climáticas; o coeficiente de cultivo $-\mathrm{K}_{\mathrm{c}}$ adotado foi de 0,8 (correspondendo à fase em que a cultura se encontrava); o coeficiente de estresse hídrico $-\mathrm{K}_{\mathrm{s}}$ foi estimado pelo modelo recomendado por Bernardo et al. (2005) e o coeficiente de localização $-\mathrm{K}_{\mathrm{l}}$ foi estimado segundo modelo proposto por Keller \& Bliesner (1990).

$$
\mathrm{Et}_{\mathrm{c}}=\mathrm{Et}_{\mathrm{o}} \mathrm{K}_{\mathrm{c}} \mathrm{K}_{\mathrm{s}} \mathrm{K}_{1}
$$

Com base na $\operatorname{ET}_{\mathrm{c}}$ (Figura 2) e na eficiência de aplicação do sistema no tempo $1-\mathrm{T}_{\mathrm{p} 1}$ (após 90 dias da adoção dos manejos) aplicou-se uma lâmina de irrigação suplementar acumulada de $46 \mathrm{~mm}$; no tempo 2 - $\mathrm{T}_{\mathrm{p} 2}$ (após 180 dias da adoção dos manejos) a lâmina de irrigação suplementar acumulada totalizou $55 \mathrm{~mm}$ e, no tempo 3 - $\mathrm{T}_{\mathrm{p} 3}$ (após 270 dias da adoção dos manejos) a lâmina de irrigação suplementar acumulada foi de $101 \mathrm{~mm}$.

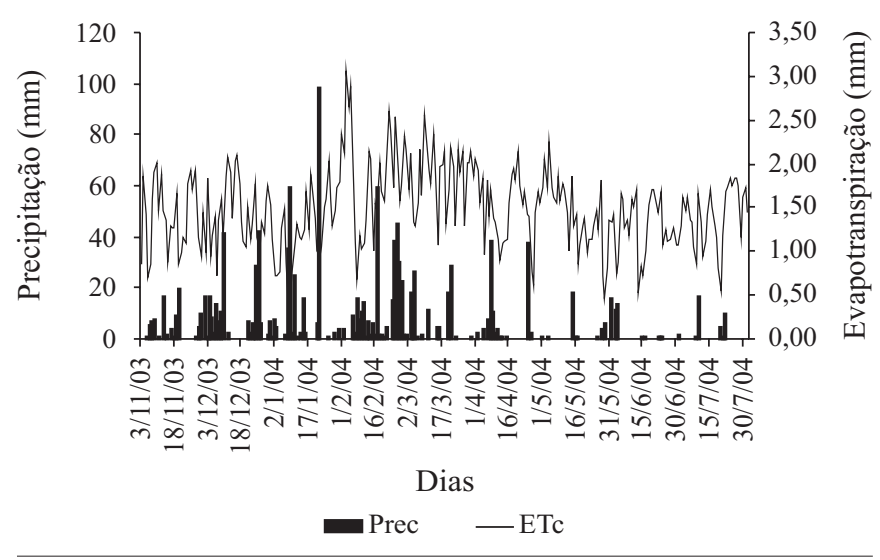

Figura 2. Evapotranspiração do cafeeiro e precipitação registrada durante o período de monitoramento

No MR o critério adotado na definição das lâminas de água residuária de origem doméstica baseou-se no aporte de $\mathrm{N}$ - total ao solo; as lâminas aplicadas teriam de aportar o equivalente a 200, 300, 400, 500 e $600 \mathrm{~kg} \mathrm{ha}^{-1} \mathrm{ano}^{-1}$ de $\mathrm{N}$ - total ao solo, aos tratamentos $\mathrm{T}_{2}, \mathrm{~T}_{3}, \mathrm{~T}_{4}, \mathrm{~T}_{5}$ e $\mathrm{T}_{6}$, respectivamente; contudo, as lâminas a serem aplicadas totalizariam 401, 600, 802, 1001 e $1201 \mathrm{~mm}^{\text {ano-1 }}$, tendose uma concentração média de $\mathrm{N}$ - total na água residuária de $50 \mathrm{mg} \mathrm{L}^{-1}$; todavia, em razão das precipitações ocorridas no período (Figura 2), as lâminas de água residuária aplicadas acumuladas totalizaram: 117, 146, 234, 264 e
$293 \mathrm{~mm}$ no $\mathrm{T}_{\mathrm{p} 1}$, no $\mathrm{T}_{\mathrm{p} 2} 155,197,309,360$ e $399 \mathrm{~mm}$ e no $\mathrm{T}_{\mathrm{p} 3}$ 202, 262, 399, 468 e $532 \mathrm{~mm}$.

A freqüência da aplicação da água residuária foi diária, exceto aos sábados e domingos; para se estimar os aportes de nutrientes às plantas, por meio das diferentes lâminas de água residuária aplicadas a cada irrigação, foi coletada uma alíquota (100 mL) de água, armazenando-as em freezer; ao final de cada mês, fez-se uma amostra composta, para determinação de $\mathrm{pH}$, condutividade elétrica - CE, concentrações de N, P, K, Ca, Mg, Zn, Cu, Fe e Mn, conforme metodologia proposta pela APHA (1997).

Os dados foram analisados para cada tempo $\left(\mathrm{T}_{\mathrm{p} 1}, \mathrm{~T}_{\mathrm{p} 2}\right.$, e $\mathrm{T}_{\mathrm{p} 3}$ ), em função do tipo de manejo adotado (MC e MR); de início, realizou-se a análise de variância de cada nutriente monitorado em cada tempo; posteriormente, verificou-se a influência das diferentes lâminas de água residuária aplicadas no estado nutricional do cafeeiro em cada tempo; todavia, na grande maioria dos casos (88\%), não se observaram diferenças significativas nos teores foliares do cafeeiro, em função da lâmina aplicada. Ante esses resultados, deuse prioridade por realizar uma estatística descritiva dos teores foliares do cafeeiro e testar um contraste (com o nível máximo de significância de 5\% de probabilidade) para verificar os efeitos médios nos teores foliares do cafeeiro, em cada tempo e em resposta à adoção dos manejos com água residuária e convencional. Para as análises estatísticas utilizando-se o software SAEG 5.0 (FUNARBE, 1993).

\section{RESULTADOS E DISCUSSÃO}

Apresenta-se, na Tabela 2, a estimativa de aporte de nutrientes à cultura do cafeeiro, via fertirrigação, durante o período de monitoramento. De acordo com as características iniciais do solo (Tabela 1) e as exigências nutricionais do cafeeiro, verificou-se que a aplicação da água residuária filtrada de origem doméstica começou a atender às exigências de $\mathrm{P}$ no $\mathrm{T}_{\mathrm{p} 2}$ com aplicação de uma lâmina de 197 mm; todavia, a aplicação de K e N ficaram 72,6 e 72,2\%, respectivamente, aquém do necessário. Em relação aos micronutrientes ( $\mathrm{Zn}, \mathrm{Cu}$ e $\mathrm{Mn}$ ), apenas no $\mathrm{T}_{\mathrm{p} 3}$ a aplicação da maior lâmina $\left(\mathrm{T}_{6}\right)$ supriria a necessidade de $\mathrm{Cu}$, considerando-se um solo com baixa disponibilidade.

A Tabela 3 aponta as concentrações de nutrientes detectadas nas folhas do cafeeiro, no início do experimento e durante o período de monitoramento. Observa-se que, antes da adoção dos manejos, o cafeeiro apresentava concentrações de N, K, Ca e Mg abaixo da faixa de valores considerados críticos, em folhas do cafeeiro, e as concentrações de S, Zn, Fe e Mn estavam acima, e somente as concentrações de $\mathrm{P}$, Cu e B estavam dentro da faixa recomendada, evidenciando que o cafeeiro apresentava distúrbios nutricionais antes da adoção dos manejos, fato este influenciado pelas características iniciais do solo (acidez elevada, concentração de P disponível considerada muito baixa e concentrações de $\mathrm{K}^{+}, \mathrm{Ca}^{2+}$ e $\mathrm{Mg}^{2+}$ trocáveis, baixas).

Verificou-se que a adoção dos manejos influenciou diretamente nas concentrações de nutrientes nas folhas do cafeeiro 
Tabela 2. Aporte de nutrientes aplicados à cultura do cafeeiro via adubação convencional e água residuária, durante o período de monitoramento

\begin{tabular}{|c|c|c|c|c|c|c|c|c|c|c|c|}
\hline \multirow{2}{*}{\multicolumn{2}{|c|}{$\begin{array}{c}\text { Lâminas } \\
\text { Tratamentos acumuladas } \\
(\mathrm{mm})\end{array}$}} & $P$ & K & N-Total & $\mathrm{Na}$ & $\mathrm{Ca}$ & $\mathrm{Mg}$ & $\mathrm{Zn}$ & $\mathrm{Cu}$ & $\mathrm{Fe}$ & $\mathrm{Mn}$ \\
\hline & & \multicolumn{10}{|c|}{$\left(\mathrm{kg} \mathrm{ha}^{-1}\right)$} \\
\hline & & \multicolumn{10}{|c|}{ Tempo $1\left(\mathrm{~T}_{\mathrm{p} 1}\right)$} \\
\hline $\mathrm{T}_{1}$ & - & 21,43 & 248,94 & 300,00 & - & - & - & - & - & - & - \\
\hline $\mathrm{T}_{2}$ & 117 & 7,76 & 41,30 & 29,49 & 62,18 & 12,16 & 3,18 & 0,16 & 1,03 & 8,66 & 0,19 \\
\hline $\mathrm{T}_{3}$ & 146 & 9,74 & 51,46 & 36,85 & 77,54 & 15,55 & 3,97 & 0,21 & 1,42 & 11,48 & 0,25 \\
\hline $\mathrm{T}_{4}$ & 234 & 15,53 & 82,59 & 58,99 & 124,34 & 24,32 & 6,35 & 0,32 & 2,06 & 17,32 & 0,39 \\
\hline $\mathrm{T}_{5}$ & 264 & 17,57 & 93,09 & 66,63 & 140,22 & 27,74 & 7,18 & 0,37 & 2,42 & 20,12 & 0,44 \\
\hline \multirow[t]{2}{*}{$\mathrm{T}_{6}$} & 293 & 19,53 & 103,07 & 73,92 & 155,35 & 31,16 & 7,97 & 0,42 & 2,83 & 23,07 & 0,51 \\
\hline & & \multicolumn{10}{|c|}{ Tempo $2\left(\mathrm{~T}_{\mathrm{p} 2}\right)$} \\
\hline $\mathrm{T}_{1}$ & - & - & - & - & - & - & - & - & - & - & - \\
\hline $\mathrm{T}_{2}$ & 155 & 17,63 & 53,79 & 63,26 & 80,61 & 15,29 & 4,01 & 0,17 & 1,08 & 9,88 & 0,20 \\
\hline $\mathrm{T}_{3}$ & 197 & 23,28 & 68,15 & 83,26 & 102,25 & 19,75 & 5,08 & 0,23 & 1,49 & 13,16 & 0,27 \\
\hline $\mathrm{T}_{4}$ & 309 & 35,25 & 107,25 & 126,47 & 160,74 & 30,50 & 7,99 & 0,34 & 2,16 & 19,75 & 0,41 \\
\hline $\mathrm{T}_{5}$ & 360 & 41,56 & 124,20 & 149,43 & 186,55 & 35,52 & 9,22 & 0,40 & 2,56 & 23,30 & 0,47 \\
\hline \multirow[t]{2}{*}{$\mathrm{T}_{6}$} & 399 & 46,86 & 137,97 & 167,66 & 206,99 & 39,89 & 10,28 & 0,45 & 2,97 & 26,52 & 0,54 \\
\hline & & \multicolumn{10}{|c|}{ Tempo $3\left(\mathrm{~T}_{\mathrm{p} 3}\right)$} \\
\hline $\mathrm{T}_{1}$ & - & - & - & - & - & - & - & - & - & - & - \\
\hline $\mathrm{T}_{2}$ & 202 & 20,86 & 66,57 & 80,54 & 92,93 & 25,87 & 6,17 & 0,19 & 1,13 & 11,99 & 0,27 \\
\hline $\mathrm{T}_{3}$ & 262 & 27,70 & 85,58 & 106,92 & 119,09 & 34,22 & 8,03 & 0,25 & 1,56 & 16,07 & 0,36 \\
\hline $\mathrm{T}_{4}$ & 399 & 41,41 & 131,60 & 159,44 & 184,21 & 50,65 & 12,11 & 0,38 & 2,26 & 23,77 & 0,54 \\
\hline$T_{5}$ & 468 & 49,02 & 153,59 & 189,43 & 214,93 & 59,89 & 14,20 & 0,45 & 2,68 & 28,20 & 0,63 \\
\hline $\mathrm{T}_{6}$ & 532 & 56,03 & 174,14 & 216,89 & 241,90 & 69,88 & 16,42 & 0,51 & 3,13 & 32,55 & 0,73 \\
\hline
\end{tabular}

Tabela 3. Estado nutricional do cafeeiro em resposta à adoção dos tipos de manejo

\begin{tabular}{|c|c|c|c|c|c|c|c|c|c|c|c|c|}
\hline \multirow[b]{3}{*}{$\mathrm{FC}^{*}$} & & $\mathrm{~N}$ & $P$ & K & $\mathrm{Ca}$ & $\mathrm{Mg}$ & $S$ & $\mathrm{Zn}$ & $\mathrm{Fe}$ & $\mathrm{Mn}$ & $\mathrm{Cu}$ & B \\
\hline & & \multicolumn{6}{|c|}{$\mathrm{g} \mathrm{kg}^{-1}$} & \multicolumn{5}{|c|}{$\mathrm{mg} \mathrm{kg}^{-1}$} \\
\hline & & $26,4-30,8$ & $2,2-2,6$ & $21,8-28,4$ & $12,1-14,5$ & $3,4-5,8$ & $1,0-1,2$ & $6,0-12,0$ & $62,0-88,0$ & $94,0-313,0$ & $12,0-29,0$ & $28,0-52,0$ \\
\hline \multirow[t]{3}{*}{$\mathrm{C}_{\mathrm{ic}}$} & & 24,90 & 2,24 & 12,00 & 7,82 & 2,66 & 1,46 & 12,40 & 132,00 & 365,30 & 22,50 & 46,80 \\
\hline & & \multicolumn{11}{|c|}{ Tempo $1\left(T_{p 1}\right)$} \\
\hline & M & 29,40 & 1,37 & 15,80 & 8,53 & 3,27 & 1,63 & 8,57 & 49,33 & 237,23 & 9,03 & 20,93 \\
\hline \multirow[t]{3}{*}{$M C^{1}$} & $\mathrm{DP}$ & 15,74 & 0,06 & 0,69 & 0,64 & 0,21 & 0,21 & 0,38 & 5,81 & 76,75 & 1,25 & 5,36 \\
\hline & IC & 17,82 & 0,07 & 0,78 & 0,73 & 0,24 & 0,24 & 0,43 & 6,58 & 86,84 & 1,41 & 6,06 \\
\hline & $\mathrm{M}$ & 27,63 & 1,80 & 11,71 & 12,45 & 3,43 & 1,26 & 8,67 & 67,45 & 757,06 & 10,77 & 50,84 \\
\hline \multirow[t]{4}{*}{$\mathrm{MR}^{2}$} & $\mathrm{DP}$ & 2,56 & 0,12 & 2,27 & 1,88 & 0,28 & 0,28 & 0,90 & 13,54 & 228,85 & 2,76 & 17,36 \\
\hline & IC & 1,30 & 0,06 & 1,15 & 0,95 & 0,14 & 0,14 & 0,46 & 6,85 & 115,81 & 1,39 & 8,78 \\
\hline & & \multicolumn{11}{|c|}{ Tempo $2\left(\mathrm{~T}_{\mathrm{p} 2}\right)$} \\
\hline & M & 32,03 & 1,34 & 19,01 & 8,47 & 3,61 & 1,05 & 6,00 & 109,63 & 368,97 & 13,30 & 28,83 \\
\hline \multirow[t]{3}{*}{$M C^{1}$} & $\mathrm{DP}$ & 0,51 & 0,14 & 0,30 & 0,96 & 0,30 & 0,07 & 0,80 & 18,22 & 125,56 & 2,14 & 1,88 \\
\hline & IC & 0,57 & 0,16 & 0,34 & 1,08 & 0,34 & 0,08 & 0,91 & 20,62 & 142,08 & 2,42 & 2,12 \\
\hline & M & 29,38 & 1,77 & 10,63 & 13,03 & 4,32 & 1,30 & 6,26 & 99,99 & 655,18 & 15,42 & 75,00 \\
\hline \multirow[t]{4}{*}{$\mathrm{MR}^{2}$} & $\mathrm{DP}$ & 1,82 & 0,24 & 2,66 & 2,26 & 0,35 & 0,23 & 0,99 & 27,29 & 285,93 & 3,47 & 16,67 \\
\hline & IC & 0,92 & 0,12 & 1,34 & 1,14 & 0,18 & 0,12 & 0,50 & 13,81 & 144,70 & 1,76 & 8,44 \\
\hline & & \multicolumn{11}{|c|}{ Tempo $3\left(\mathrm{~T}_{\mathrm{p} 3}\right)$} \\
\hline & $\mathrm{M}$ & 26,03 & 1,35 & 12,18 & 6,74 & 2,27 & 1,31 & 8,43 & 94,13 & 278,97 & 13,07 & 21,63 \\
\hline \multirow[t]{3}{*}{$\mathrm{MC}^{1}$} & $\mathrm{DP}$ & 0,84 & 0,09 & 3,42 & 1,17 & 0,23 & 0,06 & 0,59 & 12,41 & 88,24 & 3,27 & 7,33 \\
\hline & IC & 0,95 & 0,11 & 3,87 & 1,32 & 0,26 & 0,07 & 0,66 & 14,05 & 99,85 & 3,70 & 8,30 \\
\hline & $\mathrm{M}$ & 29,58 & 1,91 & 9,91 & 10,64 & 3,16 & 1,91 & 8,99 & 86,65 & 518,51 & 21,07 & 67,63 \\
\hline \multirow[t]{2}{*}{$\mathrm{MR}^{2}$} & $\mathrm{DP}$ & 2,93 & 0,30 & 2,22 & 1,96 & 0,60 & 0,44 & 0,58 & 11,22 & 198,95 & 9,71 & 22,87 \\
\hline & IC & 1,48 & 0,15 & 1,12 & 0,99 & 0,30 & 0,22 & 0,29 & 5,68 & 100,68 & 4,91 & 11,57 \\
\hline
\end{tabular}

*FC: Faixas críticas de macro e micronutrientes em folhas de cafeeiros (Fonte: Recomendação para Uso de Corretivos e Fertilizantes do Estado de Minas Gerais - $5^{a}$ aproximação, 1999) $\mathrm{C}_{\mathrm{ic}}$ : Condição inicial do cafeeiro (estado nutricional); MC: Manejo convencional; MR: Manejo com água residuária; M: Valor médio; DP: Desvio padrão e IC: Intervalo de confiança

1 Os valores das variáveis no manejo convencional provêm da média das 3 repetições

${ }^{2} 0$ s valores das variáveis no manejo com água residuária se originaram da média geral dos tratamentos $T_{2}, T_{3}, T_{4}, T_{5}$ e $T_{6}$ 
(Tabela 3) e que, no $T_{p 1}$, a adoção do $M C$ proporcionou um incremento nas concentrações de $\mathrm{N}, \mathrm{K}, \mathrm{Ca}, \mathrm{Mg}$ e $\mathrm{S}$ em relação à condição inicial, mas provocou decréscimos nas de $\mathrm{P}$, $\mathrm{Zn}, \mathrm{Fe}, \mathrm{Mn}$, Cu e B; já no MR ocorreu incremento nas concentrações de N, Ca, Mg, Mn e B e decréscimo nas de P, K, $\mathrm{S}, \mathrm{Zn}, \mathrm{Fe}$ e Cu. Apesar do acréscimo e do decréscimo de alguns nutrientes na folha em resposta ao tipo de manejo adotado, notou-se que no MC apenas as concentrações de N, Zn e Mn permaneceram em níveis adequados e, no $\mathrm{MR}$, as concentrações de N, Ca, Mg, Zn, Fe e B.

No $T_{p 2}$ foi notório o aumento nas concentrações de $N$, K, $\mathrm{Mg}, \mathrm{Fe}, \mathrm{Mn}, \mathrm{Cu}$ e B nas folhas, em relação ao $\mathrm{T}_{\mathrm{p} 1}$, em resposta à adoção do $\mathrm{MC}$; já no $\mathrm{MR}$ os nutrientes que tiveram incremento foram o N, Ca, Mg, S, Fe, Mn, Cu e o B (Tabela 3), mas se observou que no MC os nutrientes que permaneceram em níveis adequados foram $\mathrm{Mg}, \mathrm{S}, \mathrm{Zn}, \mathrm{Cu}$ e $\mathrm{B}$ e, no MR, as concentrações de N, Ca, Mg, S, Zn e Cu. Ao final do experimento $\left(\mathrm{T}_{\mathrm{p} 3}\right)$, o MC proporcionou aumento nas concentrações de $\mathrm{P}, \mathrm{S}$ e $\mathrm{Zn}$ nas folhas, em relação ao $\mathrm{T}_{\mathrm{p} 2}$, enquanto a adoção do MR provocou acréscimo nas concentrações de N, P, S, Zn e Cu.

Avaliando-se o incremento dos macro e micronutrientes nas folhas do cafeeiro notou-se que, na maioria dos casos, o MR foi mais efetivo no aumento da concentração que o MC (Tabela 4).

Dentre os nutrientes que mais incrementaram nas folhas do cafeeiro em resposta à adoção do $\mathrm{MR}$, destacam-se o $\mathrm{P}$, Ca e o B (Tabela 4); todavia, as concentrações de P nas folhas permaneceram abaixo dos níveis críticos, em todos os tempos avaliados; as de Ca só foram adequadas nos $\mathrm{T}_{\mathrm{p} 1} \mathrm{e}$ $\mathrm{T}_{\mathrm{p} 2}$, e baixas no $\mathrm{T}_{\mathrm{p} 3}$; já as de $\mathrm{B}$ só foram consideradas adequadas no $\mathrm{T}_{\mathrm{p} 1}$ e altas nos demais (Tabela 3 ).

Os incrementos de P, Ca e B nas folhas do cafeeiro, em resposta ao MR, foram ocasionados pela maior disponibilidade desses nutrientes no solo (Tabela 1), principalmente nas primeiras profundidades influenciadas pela aplicação de água residuária filtrada, de origem doméstica.

No que se refere à concentração de $\mathrm{N}$ nas folhas, o MC proporcionou um incremento maior no $\mathrm{T}_{\mathrm{p} 1}$ e $\mathrm{T}_{\mathrm{p} 2}$ (Tabela 3), porém só foi significativo no $\mathrm{T}_{\mathrm{p} 2}$ (Tabela 4); apesar desse incremento, a concentração de $\mathrm{N}$ na folha em resposta à adoção do MR foi considerada adequada durante todo o período monitorado (Tabela 3 ).

As concentrações de $\mathrm{K}$ nas folhas foram consideradas baixas em resposta à adoção dos dois tipos de manejo (Tabela 3), mas nos $\mathrm{T}_{\mathrm{p} 1}$ e $\mathrm{T}_{\mathrm{p} 2}$, o MC, proporcionou incrementos significativos nas concentrações de $\mathrm{K}$ nas folhas do cafeeiro, em relação ao MR (Tabela 4). Tais incrementos estão diretamente relacionados aos aumentos das disponibilidades $\mathrm{K}^{+}$no solo, ocasionado pela aplicação de cloreto de potássio e pela baixa capacidade da água residuária filtrada no suprimento desse nutriente ao cafeeiro.

Quanto às concentrações de $\mathrm{S}, \mathrm{Zn}$, Fe e $\mathrm{Cu}$ nas folhas do cafeeiro, não foram detectados incrementos significativos em relação ao tipo de manejo adotado (Tabela 4); todavia, as concentrações de $\mathrm{S}$ nas folhas foram consideradas altas, independentemente do tipo de manejo adotado, com exceção do $\mathrm{T}_{\mathrm{p} 2}$, no MC, cujas concentrações foram adequadas (Tabela 3).
Tabela 4. Diferenças médias (MR - MC) na concentração foliar do cafeeiro em resposta ao tipo de manejo adotado

\begin{tabular}{|c|c|c|c|c|}
\hline \multirow{2}{*}{$\begin{array}{c}\text { Tempo } \\
1\end{array}$} & \multirow[t]{2}{*}{ Variável } & \multirow[t]{2}{*}{ Und. } & \multicolumn{2}{|c|}{ Diferenças (MR - MC) } \\
\hline & & & $-1,77$ & $\mathrm{~ns}$ \\
\hline 2 & N & $\mathrm{g} \mathrm{kg}^{-1}$ & $-2,65$ & * \\
\hline 3 & & & 3,55 & * \\
\hline 1 & & & 0,44 & ** \\
\hline 2 & $P$ & $\mathrm{~g} \mathrm{~kg}^{-1}$ & 0,43 & * \\
\hline 3 & & & 0,56 & * \\
\hline 1 & & & $-4,09$ & * \\
\hline 2 & K & $\mathrm{g} \mathrm{kg}^{-1}$ & $-8,38$ & ** \\
\hline 3 & & & $-2,27$ & ns \\
\hline 1 & & & 3,92 & ** \\
\hline 2 & $\mathrm{Ca}$ & $\mathrm{g} \mathrm{kg}^{-1}$ & 4,56 & ** \\
\hline 3 & & & 3,90 & ** \\
\hline 1 & & & 0,17 & ns \\
\hline 2 & $\mathrm{Mg}$ & $\mathrm{g} \mathrm{kg}^{-1}$ & 0,71 & * \\
\hline 3 & & & 0,89 & * \\
\hline 1 & & & $-0,38$ & ns \\
\hline 2 & $S$ & $\mathrm{~g} \mathrm{~kg}^{-1}$ & 0,25 & ns \\
\hline 3 & & & 0,59 & ns \\
\hline 1 & & & 0,11 & ns \\
\hline 2 & $\mathrm{Zn}$ & $\mathrm{mg} \mathrm{kg}^{-1}$ & 0,26 & ns \\
\hline 3 & & & 0,55 & ns \\
\hline 1 & & & 18,12 & ns \\
\hline 2 & $\mathrm{Fe}$ & $\mathrm{mg} \mathrm{kg}^{-1}$ & $-9,64$ & ns \\
\hline 3 & & & $-7,48$ & ns \\
\hline 1 & & & 519,83 & ** \\
\hline 2 & $\mathrm{Mn}$ & $\mathrm{mg} \mathrm{kg}^{-1}$ & 286,21 & ns \\
\hline 3 & & & 239,55 & ns \\
\hline 1 & & & 1,73 & ns \\
\hline 2 & $\mathrm{Cu}$ & $\mathrm{mg} \mathrm{kg}^{-1}$ & 2,12 & ns \\
\hline 3 & & & 8,01 & ns \\
\hline 1 & & & 29,91 & * \\
\hline 2 & B & $\mathrm{mg} \mathrm{kg}^{-1}$ & 46,17 & $\star *$ \\
\hline 3 & & & 46,00 & ** \\
\hline
\end{tabular}

As concentrações de $\mathrm{Zn}$ e $\mathrm{Cu}$ nas folhas do cafeeiro foram adequadas durante todo o período de monitoramento, em resposta à adoção dos manejos, exceto no $\mathrm{T}_{\mathrm{p} 1}$, em resposta à adoção do $\mathrm{MC}$, em que a concentração de $\mathrm{Cu}$ foi considerada baixa (Tabela 3).

Por sua vez, as concentrações de Fe nas folhas do cafeeiro em resposta à adoção do MR foram tidas como adequadas aos $\mathrm{T}_{\mathrm{p} 1}$ e $\mathrm{T}_{\mathrm{p} 3}$, e altas no $\mathrm{T}_{\mathrm{p} 2}$ (Tabela 3). Com relação ao $\mathrm{MC}$, as concentrações foram consideradas altas nos $T_{\mathrm{p} 2}$ e $T_{\mathrm{p} 3}$ e baixas no $\mathrm{T}_{\mathrm{p} 1}$ (Tabela 3). As baixas concentrações de Fe nas folhas do cafeeiro verificadas no MC, podem ter sido influenciadas pela calagem, que incrementou o $\mathrm{pH}$ do solo, cuja conseqüência foi a redução da sua disponibilidade.

Verificou-se que o MR propiciou incremento maior nas concentrações de $\mathrm{Mn}$ nas folhas do cafeeiro que o MC; contudo, os incrementos foram significativos no $\mathrm{T}_{\mathrm{p} 1}$ (Tabela 4) e, 
de modo geral, as concentrações de Mn nas folhas só foram adequadas no $\mathrm{MC}$ e no $\mathrm{T}_{\mathrm{p} 1}$ (Tabela 3); nos demais tempos, ficaram acima. No MR as concentrações foram superiores ao recomendado em todo o período monitorado, mas se espera que, com a aplicação prolongada da água residuária no solo, se possa incrementar ainda mais o $\mathrm{pH}$ do solo apresentando, como efeito, redução da disponibilidade de Mn no solo.

Vários estudos demonstraram que a aplicação de água residuária elevou a concentração de macro e micronutriente nas folhas das culturas cultivadas (Feigin et al., 1978; Fitzpatrick et al., 1986; Maurer \& Davies, 1993; Al-Jaloud et al., 1995; Sandri, 2003; Rezende, 2003) e que, de modo geral, este aumento é influenciado pela concentração de nutrientes na água. Com o exposto, verifica-se que a adoção do MR pode vir como alternativa de fertilização das culturas, tendo em vista o seu excelente desempenho no suprimento de alguns nutrientes.

\section{CONCLUSÕES}

1. A adoção dos manejos não foram efetivos para elevar as concentrações dos macro e micronutrientes nas folhas, a níveis considerados adequados para a cultura do cafeeiro, porém se verificou que a adoção do $\mathrm{MR}$ foi mais efetiva na melhoria do estado nutricional do cafeeiro que o MC, revelando-se que a aplicação controlada de água residuária ao solo pode vir como alternativa para fertilização das culturas, potencializando a produção de alimentos; contudo, é conveniente o monitoramento constante dos atributos do solo, a fim de se identificar possíveis contaminações decorrentes da aplicação de água residuária.

2. Outros aspectos que não foram enfocados neste trabalho e devem ser estudados a fim de potencializar definitivamente a produção de alimentos (exceto os consumidos crus, principalmente olericolas) utilizando-se a água residuária, se refere à contaminação microbiológica dos alimentos produzidos e aos riscos potenciais de saúde pública, inerentes a esta atividade.

\section{LITERATURA CITADA}

Al-Jaloud, A. A.; Hussain, G.; Al-Saati, A. J.; Karimulla, S. Effect of wastewater irrigation on mineral composition of corn and sorghum plants in a pot experiment. Journal of Plant Nutrition, v.18, n.8, p.1677-1692, 1995.

Allen, R. G.; Pereira, L. S.; Raes, D.; Smith. M. Crop evapotranspiration - Guidelines for computing crop water requirements. In: FAO Irrigation and Drainage Paper 56. Rome: FAO, 1998. 308p.

APHA - American Public Health Association. Standard methods for the examination of water and wastewater. 20.ed. New York: APHA, AWWA, WPCR, 1997. 1194p.

Bernardo, S.; Soares, A. A.; Mantovani, E. C. Manual de irrigação. 7.ed. Viçosa: UFV, 2005. 611p.
Cameron, K. C.; DI, H. J.; Mclaren, R. G. Is soil an appropriate dumping ground for our wastes. Australian Journal of Soil Research, v.35, n.5, p.995-1035, 1997.

Chae, Y. M.; Tabatabai, M. A. Mineralization of nitrogen in soils amended with organic wastes. Journal of Environmental Quality, v.15, n.2, p.193-198, 1986.

CFSEMG - Comissão de Fertilidade do Solo do Estado de Minas Gerais. Recomendações para o uso de corretivo e fertilizantes em Minas Gerais. - 5a aproximação. In: Ribeiro, A. C.; Guimarães, P. T. G; Alvares V. V. H. (eds.). Viçosa. 1999, 359p.

EMBRAPA - Empresa Brasileira de Pesquisa Agropecuária. Manual de métodos de análise do solo. 2.ed. Rio de Janeiro: Embrapa CNPS, 1997. 247p.

Feigin, A.; Bielorai, H.; Dag, Y.; Kipnis, T.; Giskin, M. The nitrogen factor in the management of effluent- irrigated soils. Soil Science, v.125, n.4, p.248-254, 1978.

Fitzpatrick, G.; Donselman, H.; Carter, N. S. Interactive effects of sewage effluent irrigation and supplemental fertilization on container-grown trees. HortScience, v.21, n.1, p.92-93, 1986.

FUNARBE. SAEG - Sistema para análises estatísticas - versão 5.0. Viçosa: Fundação Arthur Bernardes. 1993. 80p.

Johns, G. G.; McConchie, D. M. Irrigation of bananas with secondary treated sewage effluent. I. Field evaluation of effect on plant nutrients and additional elements in leaf, pulp and soil. Australian Journal of Agricultural Research, v.45, n.7, p.1601-1617, 1994a.

Johns, G. G.; McConchie, D. M. Irrigation of bananas with secondary treated sewage effluent. II. Effect on plant nutrients, additional elements and pesticide residues in plants, soil and leachate using drainage lysimeters. Australian Journal of Agricultural Research, v.45, n.7, p.1619-1638, 1994b.

Keller, J.; Bliesner, R. D. Sprinkler and trickle irrigation. New York: Avibook, 1990. 649p.

Lurie, S.; Zilkah, S.; David, I.; Lapsker, Z.; Arie, R. B. Quality of "Flamekist" nectarine fruits from on orchard irrigated with reclaimed sewage water. Journal of Horticultural Science, v.71, p.313-319, 1996.

Maurer, M. A.; Davies, F. S. Microsprinkler irrigation of young "Redblush" grapefruit trees using reclaimed water. HortScience, v.28, n.12, p.1157-1161, 1993.

Rezende, A. A. P. Fertirrigação do eucalipto com efluente tratado de fábrica de celulose kraft branqueada. Viçosa: UFV. 2003, 152p. Tese Doutorado

Sandri, S. Irrigação da cultura da alface com água residuária tratada com leitos cultivados com macrófita. Campinas: UNICAMP. 186p, 2003. Tese Doutorado

van der Hoek, W.; Hassan, U. M.; Ensink, J. H. J.; Feenstra, S.; Raschid-Sally, L.; Munir, S.; Aslam, R.; Alim, N.; Hussain, R.; Matsuno, Y. Urban Wastewater: A valuable resoure for agriculture. a case study from horoonabad, Pakistan. Research Report 63. Colombo, Sri Lanka: International Water Management Institute. 2002. 20p.

Vieira, E. O. Índices de lixiviação e modelagem do transporte de pesticidas no solo. Viçosa: UFV. 184p. 2003. Tese Doutorado 